\title{
An in vitro study examining a novel suction curette device for lumbar discectomy compared with standard manual discectomy
}

\author{
William F. Lavelle, MD, ${ }^{1}$ Nathaniel R. Ordway, MS, ${ }^{1}$ Ali Araghi, DO, ${ }^{2}$ Rudolph A. Buckley, MD, ${ }^{3}$ and \\ Amir H. Fayyazi, MD ${ }^{4}$ \\ ${ }^{1}$ Department of Orthopedic Surgery, State University of New York Upstate Medical University, Syracuse, New York; ${ }^{2}$ The CORE \\ Institute, Phoenix, Arizona; ${ }^{3}$ Hamilton Orthopaedic Surgery \& Sports Medicine, Hamilton, New York; and ${ }^{4}$ OAA Orthopaedic \\ Specialists, Allentown, Pennsylvania
}

\begin{abstract}
OBJECTIVE This purpose of this study was to objectively evaluate and assess the efficacy and efficiency of discectomy and endplate preparation during transforaminal lumbar interbody fusion (TLIF) using traditional manual instrumentation versus a novel suction discectomy curette. Transforaminal lumbar interbody fusion is the most widely used approach for lumbar arthrodesis, and its success depends on the ability to achieve fusion. Complete preparation of intervertebral disc space (removal of the nucleus, endplate cartilage, and margin of inner annulus) is the surgical goal. Performing an adequate discectomy requires numerous instrument passes, increasing surgical time and the risk of complications.
\end{abstract}

METHODS Four experienced spinal surgeons performed transforaminal discectomies from T-12 to S-1 on 5 whole-body cadavers. Each level $(n=26)$ was randomly assigned to either a control group using traditional instruments (12 levels) or to a suction curette group (14 levels). The time required to perform the discectomy and the number of passes through the annulus were recorded. Motion segments were dissected and analyzed by digital photogrammetric analysis. The intervertebral disc and the discectomy cross-sectional areas were measured on both superior and inferior images of each dissected surgical level. Areas were divided into 4 quadrants based on a midsagittal and midcoronal axis and analyzed for regional efficiency. In addition, a cross-sectional area of bony endplate (the area still covered with cartilage) and an area of endplate perforation were evaluated.

RESULTS There was no significant difference in surgical time between the techniques $(7: 51 \pm 2: 43$ minutes in the manual discectomy [MD] group and 7:06 $\pm 3: 33$ minutes in the suction curette discectomy [SD] group). There were significantly fewer $(p<0.01)$ instrument passes in the SD group (13 passes) compared with the MD group (43 passes). For both techniques, the amount of disc removed depended upon the anatomical region, with the posterior-contralateral side having the least amount of disc material removed. There was significantly less $(p<0.01)$ disc material removed in the MD group (38\%) compared with the SD group (48\%). The amount of disc material removed was significantly more ( $p$ $<0.05$ ) in each quadrant when comparing the SD and MD groups, with the anterior regions showing the largest difference. For both techniques, the preparation of the endplate within the discectomy area resulted in a mostly cartilaginous interface (50\% MD, $48 \% \mathrm{SD})$; a smaller amount of bony interface area $(31 \% \mathrm{MD}, 38 \% \mathrm{SD})$; and a smaller amount of perforation to the interface area (19\% MD, 13\% SD). There were no significant differences between the groups in terms of endplate preparation.

CONCLUSIONS The improved discectomy observed with the suction curette device could potentially improve the clinical fusion rate.

https://thejns.org/doi/abs/10.3171/2016.9.SPINE16283

KEY WORDS transforaminal lumbar interbody fusion; TLIF; manual discectomy; suction curette discectomy

$\mathrm{T}$ Ransforaminal lumbar interbody fusion (TLIF) is arguably the most widely used approach for lumbar interbody arthrodesis. ${ }^{3,7}$ It was initially described by Harms in the early 1980s., ${ }^{1,2}$ The TLIF method has become popular for achieving interbody fusion, in part by limiting the need for a second anterior approach and avoiding the risks associated with anterior lumbar surgery.

There are concerns, however, about the amount of disc material that can be removed posteriorly. The ability to access the disc space posteriorly is limited by the presence

ABBREVIATIONS CSA = cross-sectional area; $M D=$ manual discectomy; $S D=$ suction curette discectomy; $T L I F=$ transforaminal lumbar interbody fusion . SUBMITTED March 9, 2016. ACCEPTED September 12, 2016.

INCLUDE WHEN CITING Published online January 27, 2017; DOI: 10.3171/2016.9.SPINE16283. 
of the lumbar nerve roots. Limited access does reduce the ability to adequately prepare the disc space for a fusion. Complete preparation of the intervertebral disc space includes removal of the nucleus, endplate cartilage, and a margin of the inner annulus. The removal of endplate cartilage exposes the underlying bleeding bony endplate. This allows the best potential for fusion; however, surgeons must avoid gross violation of the endplate because this may result in cage subsidence. Intraoperative assessment of the disc preparation is limited by the available surgical exposure, and, in many cases, the extent of the disc space preparation is unknown.

Inadequate removal of disc material and cartilaginous endplate increases the risk of pseudarthrosis. $\mathrm{Li}$ et $\mathrm{al}^{5}{ }^{5}$ showed that nucleus pulposus mixed with bone graft slows the rate of bony growth, possibly through inflammatory mediators. Lowe et al. ${ }^{6}$ asserted that total area of discectomy and endplate preparation play a role in fusion rate. Therefore, optimizing the potential for fusion involves maximizing intervertebral disc space and bony endplate preparation.

A thorough discectomy posteriorly requires numerous instrument passes, which can increase surgical time and risk of nerve injury or dural tear. There has been significant clinical interest in optimizing the discectomy technique by improving the efficiency of surgical instruments used for the procedure. The purpose of this study was to objectively evaluate and assess the efficacy and efficiency of discectomy and endplate preparation during TLIF using traditional instrumentation versus a novel suction discectomy curette.

\section{Methods}

\section{Specimen Preparation}

The T12-S1 levels from 5 whole-body cadavers (age at death 36-77 years, 4 male and 1 female) were used in this study. Specimens were screened with radiography (anteroposterior and lateral), dual-energy x-ray absorptiometry (DEXA), and gross examination after removal of soft tissue (preserving the intervertebral discs and all ligamentous structures). DEXA was performed on a $\mathrm{Lu}-$ nar DPXIQ Pencil Beam Densitometer (General Electric), and bone mineral density measurements were acquired using a lumbar protocol. Specimens were chosen based on the following inclusion criteria: no prior spinal surgery or instrumentation; free of osteophytes; and no significant degeneration (disc height collapse with a limit of $5 \mathrm{~mm}$ ), spinal deformity, or osteoporosis. Collapsed and fused discs were excluded.

\section{Surgical Technique}

Four experienced fellowship-trained spinal surgeons performed the procedures. These surgeons were all from different spinal surgery practices ranging from private practice to academic tertiary care practices, were trained at different fellowships, and had $>6$ years of experience as practicing spine surgeons. Each surgeon performs on average $>100$ TLIF procedures per year.

The surgical levels of each cadaver were randomized across the 4 surgeons. Randomization was completed in- dependently for L5-S1 levels and for the additional lumbar levels. Surgeons were assigned technique and level based on this randomization and were not allowed to convert techniques. Transforaminal discectomy on each level was based on each surgeon's standard technique used in their clinical practice. All of the procedures involved removal of the trailing lamina, pars, and inferior articular process of the cranial vertebra. This was followed by skeletonization of the ipsilateral pedicle of the caudal vertebra and additional laminectomy of the leading edge of the caudal vertebra using Kerrison rongeurs. The traversing nerve was then retracted, with the exiting nerve also identified for each operative level. Each surgeon then created an annulotomy using a No. 15 blade scalpel.

Disc space preparation was divided into 2 techniques. Each level was randomly assigned to either a manual (control level) discectomy group (MD) using traditional instruments or to a suction curette discectomy group (SD). Surgeons performed manual (control level) discectomies using a combination of straight and curved curettes, double-angled curettes, endplate shavers, and pituitary rongeurs in accordance with their typically used disc preparation technique. Surgeons used the novel suction curette for discectomies by scraping the endplates with suction applied through the device.

The X-tool (Ouroboros Medical, Inc.) discectomy device functions as a suction curette that simultaneously cuts and evacuates target disc tissue (Fig. 1 upper). The device has a cutter at the tip, with different angles, that uses a ring of serrated teeth to cut the nucleus, endplate cartilage, and inner annulus. A guard distal to the cutter makes the tip blunt to prevent puncture of the annulus and limits tissue entering the cannula shaft to prevent clogging (Fig. 1 lower). Standard wall suction transports strips of cut disc tissue from the distal cannula near the cutter into a clear handle that collects tissue, similar to a suction canister.

The technique of the device is similar to that of a push curette, where long push strokes with pressure against the endplates allow efficient cutting of the nucleus and endplate cartilage, and similarly, when pushed along the annulus, cut the inner annulus. The cutter can also be laterally swept along the anterior annulus to remove anterior

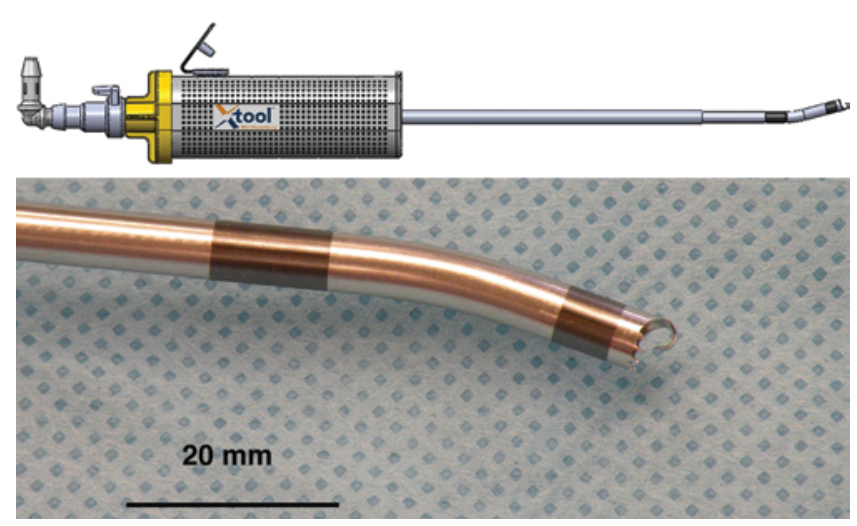

FIG. 1. Upper: The X-tool discectomy device used for this study. Lower: A close-up view of the device tip. Figure is available in color online only. 
inner annulus. In a TLIF procedure, axially covering the ipsilateral (with a $15^{\circ}$ tip) and contralateral (with a $30^{\circ}$ or $40^{\circ}$ tip) surfaces of both endplates enables a complete discectomy. The device tip is fixed, and changing the device is required to allow for the varied tip angles. The device maker was a funding source for the current study.

All surgeons completed 2 trial cases immediately prior to beginning the experiment. Standard instruments were not allowed to be used for the suction curette component of the trial.

\section{Data Analysis}

The time required to perform the discectomy and the number of passes through the annulus were recorded. The motion segments were then dissected and analyzed by digital photogrammetric analysis. The intervertebral disc and the discectomy cross-sectional areas (CSAs) were measured for both the superior and inferior images of each dissected surgical level. The areas were divided into 4 quadrants based on a midsagittal and a midcoronal axis (Fig. 2). The discectomy CSA was further analyzed by determining the CSA of bony endplate, the area still covered with cartilage, and the area of endplate perforation (exposed subchondral bone). Evaluation and complete analysis of the specimens and data were conducted by the primary authors at State University of New York Upstate Medical University and independent of the manufacturer of the suction curette device.

\section{Results}

A total of 26 levels (12 MDs and 14 SDs) were performed. The surgical time to perform the discectomy varied from surgeon to surgeon, but the number of instrument passes was fairly consistent (Table 1). There was no significant difference in surgical time $(\mathrm{p}=0.56)$ between the techniques (7:51 $\pm 2: 43$ minutes in the MD group and 7:06

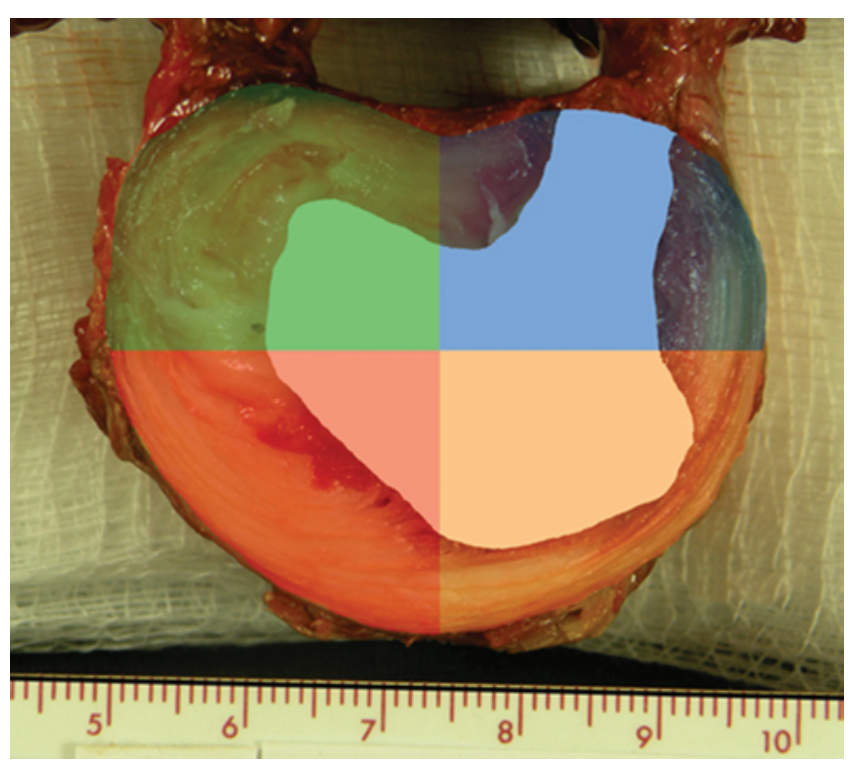

FIG. 2. Intervertebral disc CSAs divided into 4 quadrants based on midsagittal and midcoronal axes. Figure is available in color online only.
TABLE 1. Time to perform discectomy and number of instrument passes based on surgeon

\begin{tabular}{clc}
\hline Surgeon & Time $(\mathrm{sec})$ & No. of Passes \\
\hline 1 & $287 \pm 95$ & $27 \pm 17$ \\
\hline 2 & $349 \pm 89$ & $23 \pm 13$ \\
\hline 3 & $502 \pm 183$ & $32 \pm 21$ \\
\hline 4 & $608 \pm 156$ & $24 \pm 15$ \\
\hline Group & $447 \pm 189$ & $27 \pm 17$ \\
\hline
\end{tabular}

Values are reported as the mean \pm standard deviation.

$\pm 3: 33$ minutes in the SD group). Significantly fewer ( $p<$ 0.001 ) instrument passes were recorded in the SD group (13 \pm 6 instrument passes) compared with the MD group (43 \pm 9 instrument passes).

The average amount of disc material removed was $43 \%$ for all levels. For both techniques, the amount of disc removed was dependent upon the region of removal (anterior-contralateral, anterior-ipsilateral, posterior-contralateral, or posterior-ipsilateral). The smallest percentage of disc material removed was on the contralateral side $(32 \%$ posterior, $34 \%$ anterior) compared with the ipsilateral side (52\%). The most consistent amount of disc material removed across surgeons was at the anterior-contralateral location (Table 2).

There was significantly less $(\mathrm{p}<0.01)$ disc material removed in the MD group $(38 \% \pm 8 \%)$ than in the SD group $(48 \% \pm 11 \%)$. The amount of disc material removed was significantly more $(\mathrm{p}<0.05)$ in each quadrant when comparing the SD and MD groups, with the anterior regions showing the largest difference (Fig. 3).

The analysis of the endplate area by surgeon is shown in Table 3. Overall the cartilaginous, bony, and perforated endplate areas were $3.82 \mathrm{~cm}^{2}, 2.97 \mathrm{~cm}^{2}$, and $1.14 \mathrm{~cm}^{2}$, respectively, and these areas varied by surgeon. Of the 52 endplates examined (superior and inferior from 26 discs), $12(23 \%)$ displayed no perforation through the bony endplate. For both techniques, the preparation of the endplate within the discectomy area resulted in a mostly cartilaginous interface area (50\% MD, 48\% SD), a smaller amount of bony interface area (31\% MD, 38\% SD), and a smaller amount of perforation to the interface area $(19 \% \mathrm{MD}, 13 \%$ SD). There were 6 of 24 and 6 of 28 endplate perforations for the manual and suction curette techniques, respectively. Although there were greater cartilaginous and bony areas for the suction curette technique, the overall area was greater and therefore no significant differences between techniques were observed for the endplate preparation. Table 4 details the endplate areas by technique.

\section{Discussion}

Ideally, the preparation of the graft-endplate interface should be as large as possible, have bony apposition, and be mechanically stable to promote fusion and limit subsidence. To our knowledge, there are no clinical studies that have documented the relationship between the intervertebral disc space preparation and clinical outcome. There are, however, studies suggesting that successful fusion does bode well for long-term outcomes. ${ }^{4}$ 
TABLE 2. Percentage of disc material removed based on disc CSA, by location and surgeon

\begin{tabular}{cccccc}
\hline Surgeon & Anterior-Contralateral (\%) & Anterior-Ipsilateral (\%) & Posterior-Contralateral (\%) & Posterior-Ipsilateral (\%) & Total (\%) \\
\hline 1 & $33 \pm 17$ & $44 \pm 15$ & $31 \pm 14$ & $67 \pm 11$ & $44 \pm 10$ \\
\hline 2 & $33 \pm 10$ & $37 \pm 19$ & $17 \pm 10$ & $52 \pm 12$ & $35 \pm 8$ \\
\hline 3 & $34 \pm 17$ & $33 \pm 17$ & $37 \pm 17$ & $64 \pm 7$ & $43 \pm 8$ \\
\hline 4 & $36 \pm 18$ & $41 \pm 16$ & $33 \pm 17$ & $68 \pm 10$ & $45 \pm 12$ \\
\hline Group & $34 \pm 16$ & $39 \pm 16$ & $32 \pm 16$ & $65 \pm 11$ & $43 \pm 10$ \\
\hline
\end{tabular}

Values are reported as the mean \pm standard deviation.

However, in vitro studies have shown that discectomy is not always ideal in terms of maximizing the CSA and preparing the endplate. ${ }^{3,7}$ In this study, the CSA of the discectomy ranged from $38 \%$ to $48 \%$ depending upon technique, and this was similar to the overall discectomy areas of the previously mentioned studies. The current study also confirmed, quantitatively, the difficulty of removal of material from the posterior-contralateral region for a TLIF procedure. This has also been confirmed by Rihn et al., ${ }^{9}$ who found that the posterior-contralateral quadrant was the most difficult quadrant of the disc to remove in a unilateral TLIF in both minimally invasive and open approaches. Pumberger et al., in 2 separate cadaveric studies, also found that the posterior-contralateral quadrant was the most difficult disc quadrant to prepare in a unilateral TLIF.7,8

The study by Rihn et al. had a substantially better endplate preparation rate than the current study, reporting an overall endplate preparation rate of $73 \%$ by surface area. ${ }^{9}$ Our results were more consistent with those reported by Tatsumi et al., ${ }^{10}$ who found a much lower preparation rate of $39.2 \%$ that was more consistent with our study. In our study, surgeons were asked to perform what they considered to be a typical discectomy in preparation for a TLIF. They were specifically instructed to regard the cadaveric structures and setting as if they were performing a TLIF on a living specimen. Surgeons regarding this as an experimental model may have a better ability to retract structures or might spend more time in preparation than

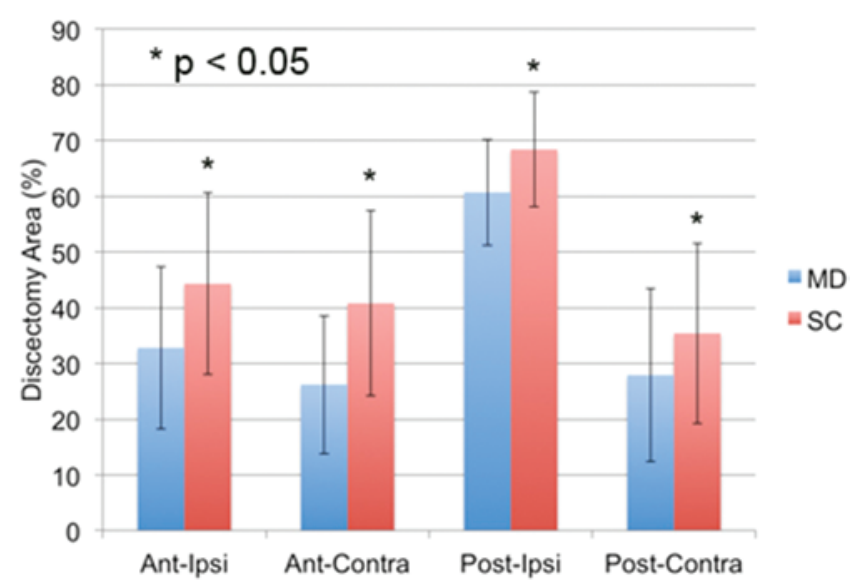

FIG. 3. Bar graph showing the comparison of SD with MD of the amount of disc material removed in each quadrant. Ant = anterior; Contra $=$ contralateral; Ipsi = ipsilateral; Post = posterior; $\mathrm{SC}$ = suction curette. Figure is available in color online only. would be typical for a living specimen. The levels where the discectomy was performed may also have had an impact on the overall surface area preparation..$^{11}$ Our study examined discs that encompassed the entire lumbar spine, as did the studies by Rihn et al. and Tatsumi et al. Neither of these studies reported the preparation rate with respect to the levels treated. Additionally, the study by Rihn et al. defined an idealized disc preparation area, whereas our study used the entire CSA of the disc space.

This simulated surgical cadaveric study also demonstrated advantages of a suction curette device over manual instruments when performing a TLIF procedure. The suction curette significantly improved the total amount of disc removed in all regions, including the posterior-contralateral region, despite the surgeons' lack of experience with the device. This improved discectomy was achieved in a similar length of time as an MD, with much fewer passes. Further evaluation of the endplate preparation did not demonstrate any significant differences between techniques regarding cartilage removal or endplate perforation. The improved discectomy seen with the suction curette device can potentially improve the clinical fusion rate.

There were limitations to the simulated surgical cadaver model. Visualization was potentially improved due to lack of intraoperative bleeding. Surgeons were asked to treat neurological structures as they would in a living patient; however, the amount of neurological retraction or manipulation cannot be verified in comparison with a living patient. The disc height cutoff for the inclusion of a cadaver level in the study was $5 \mathrm{~mm}$. This was our study protocol based on the ability to use instrumentation for disc preparation. A disc degeneration grading system would have potentially added value similar to a preoperative assessment in the clinical setting. It was not part of the protocol and is a limitation to the study. The only available imaging for the cadavers was radiography, which

TABLE 3. Endplate area analysis based on surgeon

\begin{tabular}{ccccc}
\hline Surgeon & $\begin{array}{c}\text { Cartilaginous } \\
\left(\mathrm{cm}^{2}\right)\end{array}$ & Bony $\left(\mathrm{cm}^{2}\right)$ & $\begin{array}{c}\text { Perforated } \\
\left(\mathrm{cm}^{2}\right)\end{array}$ & Total $\left(\mathrm{cm}^{2}\right)$ \\
\hline 1 & $3.85 \pm 1.95$ & $3.55 \pm 2.92$ & $0.55 \pm 0.85$ & $7.95 \pm 3.43$ \\
\hline 2 & $5.31 \pm 1.78$ & $1.48 \pm 1.14$ & $0.39 \pm 0.86$ & $7.17 \pm 1.77$ \\
\hline 3 & $4.34 \pm 1.89$ & $2.18 \pm 1.32$ & $1.41 \pm 1.64$ & $7.92 \pm 1.82$ \\
\hline 4 & $2.56 \pm 1.33$ & $3.87 \pm 2.59$ & $1.82 \pm 1.63$ & $8.25 \pm 2.57$ \\
\hline Group & $3.82 \pm 1.93$ & $2.97 \pm 2.37$ & $1.14 \pm 1.44$ & $7.93 \pm 2.55$ \\
\hline
\end{tabular}

Values are reported as the mean \pm standard deviation. 
TABLE 4. Endplate area analysis based on MD or SD

\begin{tabular}{lcccc}
\hline Technique & $\begin{array}{c}\text { Cartilaginous } \\
\left(\mathrm{cm}^{2}\right)\end{array}$ & Bony $\left(\mathrm{cm}^{2}\right)$ & $\begin{array}{c}\text { Perforated } \\
\left(\mathrm{cm}^{2}\right)\end{array}$ & Total $\left(\mathrm{cm}^{2}\right)$ \\
\hline MD & $3.37 \pm 1.53$ & $2.19 \pm 1.50$ & $1.30 \pm 1.66$ & $6.86 \pm 1.74$ \\
\hline SD & $4.20 \pm 2.16$ & $3.65 \pm 2.78$ & $1.00 \pm 1.25$ & $8.55 \pm 2.80$ \\
\hline Overall & $3.82 \pm 1.93$ & $2.97 \pm 2.37$ & $1.14 \pm 1.44$ & $7.93 \pm 2.55$ \\
\hline
\end{tabular}

Values are reported as the mean \pm standard deviation.

does limit the potential to grade disc degeneration beyond the assessment disc height and osteophyte formation.

\section{Conclusions}

A potential drawback to TLIF is adequate preparation of the intervertebral disc space in terms of the quality and quantity of the endplate interface surface for the bone graft. The improved discectomy observed with the suction curette device can potentially improve the clinical fusion rate.

\section{References}

1. Harms J, Rolinger H: [A one-stager procedure in operative treatment of spondylolistheses: dorsal traction-reposition and anterior fusion (author's transl).] Z Orthop Ihre Grenzgeb 120:343-347, 1982 (Ger)

2. Harms JG, Jeszenszky D: Die posteriore, lumbale, interkorporelle Fusion in unilateraler transforaminaler Technik. Oper Orthop Traumatol 10:90-102, 1998

3. Huh HY, Ji C, Ryu KS, Park CK: Comparison of SpineJet $\mathrm{XL}$ and conventional instrumentation for disk space preparation in unilateral transforaminal lumbar interbody fusion. J Korean Neurosurg Soc 47:370-376, 2010

4. Kornblum MB, Fischgrund JS, Herkowitz HN, Abraham DA, Berkower DL, Ditkoff JS: Degenerative lumbar spondylolisthesis with spinal stenosis: a prospective long-term study comparing fusion and pseudarthrosis. Spine (Phila Pa 1976) 29:726-734, 2004

5. Li H, Zou X, Laursen M, Egund N, Lind M, Bünger C: The influence of intervertebral disc tissue on anterior spinal interbody fusion: an experimental study on pigs. Eur Spine J 11:476-481, 2002

6. Lowe TG, Hashim S, Wilson LA, O'Brien MF, Smith DA, Diekmann MJ, et al: A biomechanical study of regional endplate strength and cage morphology as it relates to structural interbody support. Spine (Phila Pa 1976) 29:2389-2394, 2004

7. Pumberger M, Gogia J, Hughes AP, Kotwal SY, Girardi FP,
Sama AA: Conventional manual discectomy versus powered discectomy for interbody fusion in the lumbar spine: cadaveric testing in forty levels. J Spinal Disord Tech 24:E71E74, 2011

8. Pumberger M, Hughes AP, Girardi FP, Gogia J, Kotwal SY, Thaler C, et al: Influence of surgical experience on the efficiency of discectomy in TLIF: a cadaveric testing in 40 levels. J Spinal Disord Tech 25:E254-E258, 2012

9. Rihn JA, Gandhi SD, Sheehan P, Vaccaro AR, Hilibrand AS, Albert TJ, et al: Disc space preparation in transforaminal lumbar interbody fusion: a comparison of minimally invasive and open approaches. Clin Orthop Relat Res 472:18001805,2014

10. Tatsumi R, Lee YP, Khajavi K, Taylor W, Chen F, Bae H: In vitro comparison of endplate preparation between four miniopen interbody fusion approaches. Eur Spine J 24 (3 Suppl 3):372-377, 2015

11. Zheng C, Wu JJ: Disc space preparation in transforaminal lumbar interbody fusion: a comparison of minimally invasive and open approaches. Clin Orthop Relat Res 472:3243, 2014 (Letter)

\section{Disclosures}

Ouroboros Medical, Inc., partially funded this project. In addition to owning stock in Ouroboros Medical, Dr. Araghi received a one-time consulting fee from Ouroboros Medical. Dr. Fayyazi is a consultant for Ouroboros Medical. Mr. Ordway received clinical or research support for this study (includes equipment or material) from Ouroboros Medical.

\section{Author Contributions}

Conception and design: Lavelle, Ordway, Buckley, Fayyazi. Acquisition of data: Lavelle, Araghi, Buckley, Fayyazi. Analysis and interpretation of data: Lavelle, Ordway, Fayyazi. Drafting the article: Lavelle, Ordway, Fayyazi. Critically revising the article: Lavelle, Ordway, Araghi, Fayyazi. Reviewed submitted version of manuscript: all authors. Approved the final version of the manuscript on behalf of all authors: Lavelle. Statistical analysis: Lavelle, Ordway.

\section{Supplemental Information \\ Previous Presentations}

Portions of this work were presented as a poster at the American Academy of Orthopaedic Surgeons Annual Meeting, March 2014, New Orleans, LA, and at the Orthopaedic Research Society Annual Meeting, March 2015, Las Vegas, NV.

\section{Correspondence}

William F. Lavelle, Department of Orthopedic Surgery, State University of New York Upstate Medical University, 750 East Adams St., Syracuse, NY 13210. email: lavellew@upstate.edu. 